\title{
Rogue solitons in optical fibers: a dynamical process in a complex energy landscape?: supplementary material
}

\author{
Andrea Armaroli ${ }^{1,2}$, Claudio Conti ${ }^{3}$, and Fabio Biancalana ${ }^{1,4},{ }^{*}$ \\ ${ }^{1}$ Max Planck Research Group 'Nonlinear Photonic Nanostructures', Max Planck Institute for the Science of Light, \\ Günther-Scharowsky-Str. 1/Bau 2491058 Erlangen, Germany \\ ${ }^{2}$ FOTON (CNRS-UMR 6082), Université de Rennes 1, ENSSAT, 6 rue de Kerampont, CS 80518, 22305 Lannion CEDEX, France \\ ${ }^{3}$ Institute for Complex Systems (ISC-CNR) and University of Rome "La Sapienza", Department of Physics, Piazzale Aldo Moro 5 00185, \\ Rome, Italy \\ ${ }^{4}$ School of Engineering and Physical Sciences, Heriot-Watt University, EH14 4AS Edinburgh, United Kingdom \\ *Corresponding author: f.biancalana@hw.ac.uk
}

Published 14 May 2015

\begin{abstract}
This document provides supplementary information to "Rogue solitons in optical fibers: a dynamical process in a complex energy landscape?" http://dx.doi.org/10.1364/optica.2.000497, including some discussion on the fine points of the analytical and numerical techniques used therein. (c) 2015 Optical Society of America

http://dx.doi.org/10.1364/optica.2.000497.s001
\end{abstract}

\section{WEAK INTERACTION MODEL}

In order to derive the weak interaction model, we start from Eq. (1) and use the following Ansatz composed by $N$ weakly interacting NLS solitons, and model their pair-wise interaction as [1-8]

$$
\begin{aligned}
\dot{v}_{k} & =16 v_{k}^{2}\left(S_{k, k-1}-S_{k, k+1}\right) \\
\dot{\mu}_{k} & =-16 v_{k}^{2}\left(C_{k, k-1}-C_{k, k+1}\right) \\
\dot{\xi}_{k} & =2 \mu_{k}-4\left(S_{k, k-1}-S_{k, k+1}\right) \\
\dot{\delta}_{k} & =2\left(v_{k}^{2}+\mu_{k}^{2}\right)-8 \mu_{k}\left(S_{k, k-1}+S_{k, k+1}\right) \\
& +24 v_{k}\left(C_{k, k-1}+C_{k, k+1}\right)
\end{aligned}
$$

where the dot denotes the derivative with respect to $z$ and

$$
\begin{aligned}
S_{k, n} & =e^{\left|\beta_{k n}\right|} v_{n} \sin s_{k n} \phi_{k n} \\
C_{k, n} & =e^{\left|\beta_{k n}\right|} v_{n} \cos \phi_{k n} \\
\beta_{k n} & =2 v_{k}\left(\xi_{k}-\xi_{n}\right) \\
\phi_{k n} & =\delta_{k}-\delta_{n}-2 \mu_{n}\left(\xi_{k}-\xi_{n}\right)
\end{aligned}
$$

and $s_{k n}=\operatorname{sgn}\left[\beta_{k n}\right]$.

In the case of homogeneous soliton amplitudes, we recall that the WIM can be reduced to a complex Toda lattice and was further extended to the perturbed NLS, e.g. in [9], to provide a general canonical formulation; nevertheless we proved in the text that the simplest model already provides an insight on the mechanism of RS generation and perturbations such as Raman effect or higher-order dispersion are important only during collisions.

\section{NUMERICAL CALCULATION OF FIXED POINTS}

In order to find the fixed point of this system, we set $N$ and compute numerically the fixed points starting from $N_{\text {iter }}=5,000$ randomly distributed initial conditions: amplitudes $v_{k}$ are lognormally distributed, velocity $\mu_{k}$ and positions $\xi_{k}$ are normally distributed, and phases $\delta_{k}$ follow a uniform distribution in $[0,2 \pi]$.

Matlab ${ }^{\circledR}$ optimisation toolbox is employed, specifically the nonlinear solver fsolve with the Levenberg-Marquardt algorithm. Convergence is reliably achieved up to $N=40$.

\section{WEIBULL DISTRIBUTION}

Heavy-tailed distributions are a large class of PDFs which exhibit subexponential tails [10]. In mathematical form the distribution of a random variable $X$ is said to be heavy tailed if

$$
\lim _{x \rightarrow \infty} e^{\lambda t} \operatorname{Pr}[X>x]=\infty
$$

for all $\lambda>0$.

As described in the main part we fit the 95-th percentile of the peak amplitudes of the WIM fixed points with a Weibull 
distribution. We employed the Matlab®Statistics Toolbox, which implements the following probability density function (PDF)

$$
f(x \mid a, b)=b a^{-b} x^{b-1} \exp \left[-\left(\frac{x}{a}\right)^{b}\right] \theta(x),
$$

where $\theta(x)$ is the Heaviside function and $a$ and $b$ are the fitting parameters. Due to the limited sample, the frequency of rare events tends to exhibit more discrepancies with respect to the fit.

\section{ON THE SPLITTING OF NLS HAMILTONIAN DENSITY}

It is well known that the NLS Eq. (1) conserves the integrated Hamiltonian density,

$$
H(z)=\int_{-\infty}^{\infty} \mathcal{H}(z, t) \mathrm{d} t
$$

with $\mathcal{H}(z, t) \equiv \mathcal{H}_{K}+\mathcal{H}_{N L}, \mathcal{H}_{K} \equiv\left|u_{t}\right|^{2} / 2$ and $\mathcal{H}_{N L} \equiv-|u|^{4} / 2$.

We split $H(z)$ accordingly into two terms $H(z)=H_{K}(z)+$ $H_{N L}(z)$, where $H_{K}(z)$ represents the kinetic and $H_{N L}(z)$ the nonlinear (interaction) part.

In the presence of higher-order dispersion, we have to include higher-order terms in $H_{K}$, but we verified that this does not change qualitatively the conclusions reported in Fig.5.

\section{A NOTE ON RAMAN ACCELERATION}

With reference to Fig. 5(a), for a soliton with zero transverse velocity, the expected dependence of the kinetic part of the Hamiltonian is $H_{K}=H_{K}^{0}+b P_{0} Z^{2}$, where $H_{K}^{0}=\frac{1}{2} \int_{-\infty}^{\infty}\left|U_{T}\right|^{2} \mathrm{~d} T$ is the kinetic part of the NLS Hamiltonian, $P_{0}=\int_{-\infty}^{\infty}|U|^{2} \mathrm{~d} T$ is the conserved total intensity and $b=\frac{4}{15} T_{R} A^{4}$ where $T_{R}=3$ fs and $A$ is the amplitude of the soliton represents the effect of Raman acceleration. Instead we observe a jump at each collision and a subsequent steady linear increment of $H_{K}$, on account of friction-like effects due to inelastic collisions.

\section{REFERENCES}

1. G. P. Agrawal, Nonlinear Fiber Optics, 5th ed. (Academic Press, 2012).

2. I. M. Uzunov, V. S. Gerdjikov, M. Gölles, and F. Lederer, "On the description of N-soliton interaction in optical fibers," Opt. Commun. 125, 237-242 (1996).

3. V. S. Gerdjikov, D. Kaup, I. Uzunov, and E. Evstatiev, "Asymptotic behavior of N-soliton trains of the nonlinear Schrödinger equation," Phys. Rev. Lett. 77, 3943-3946 (1996).

4. D. Anderson and M. Lisak, "Bandwidth limits due to incoherent soliton interaction in optical-fiber communication systems," Phys. Rev. A 32, 2270 (1985).

5. D. Anderson and M. Lisak, "Bandwidth limits due to mutual pulse interaction in optical soliton communication systems." Opt. Lett. 11, 174-176 (1986).

6. J. M. Arnold, "Soliton pulse-position modulation," IEE Proc. J. Optoelectron. 140, 359 (1993).

7. V. I. Karpman and V. V. Solov'ev, “A perturbation theory for soliton systems," Phys. D: Nonlinear Phenomena 3, 142-164 (1981).

8. V. I. Karpman and V. V. Solov'ev, "A perturbational approach to the two-soliton systems," Phys. D: Nonlinear Phenomena 3, 487-502 (1981).
9. V. S. Gerdjikov, B. Baizakov, M. Salerno, and N. Kostov, "Adiabatic N-soliton interactions of Bose-Einstein condensates in external potentials," Phys. Rev. E 73, 046606 (2006).

10. S. Foss, D. Korshunov, S. Zachary, An Introduction to HeavyTailed and Subexponential Distributions (Springer, 2013). 